\title{
A Unifying Model-Based Hypothesis for the Diverse Waveforms of Infantile Nystagmus Syndrome
}

\author{
Zhong I. Wang \\ (1) Daroff-Dell'Osso Ocular Motility Laboratory, Louis Stokes Cleveland
} Department of Veterans Affairs Medical Center and CASE Medical School; and

(2) Department of Biomedical Engineering, Case Western Reserve University and University Hospitals Case Medical Center; Cleveland, OH, USA

\section{Louis F. Dell'Osso}

(1) Daroff-Dell'Osso Ocular Motility Laboratory, Louis Stokes Cleveland Department of Veterans Affairs Medical Center and CASE Medical School; and Departments of (2) Neurology and (3) Biomedical Engineering, Case Western Reserve University and University Hospitals Case Medical Center; Cleveland, $\mathrm{OH}, \mathrm{USA}$

\begin{abstract}
We expanded the original behavioral Ocular Motor System (OMS) model for Infantile Nystagmus Syndrome (INS) by incorporating common types of jerk waveforms within a unifying mechanism. Alexander's law relationships were used to produce desired INS null positions and sharpness. At various gaze angles, these relationships influenced the IN slow-phase amplitudes differently, thereby mimicking the gaze-angle effects of INS patients. Transitions from pseudopendular with foveating saccades to jerk waveforms required replacing braking saccades with foveating fast phases and adding a resettable neural integrator in the pursuit pre-motor circuitry. The robust simulations of accurate OMS behavior in the presence of diverse INS waveforms demonstrate that they can all be generated by a loss of pursuit-system damping, supporting this hypothetical origin.
\end{abstract}

\section{Keywords: Infantile Nystagmus Syndrome, mechanism, waveforms, model, simulation}

\section{Introduction}

Infantile Nystagmus Syndrome (INS) (CEMAS_Working_Group, 2001) may exhibit several types of either pendular or jerk wave-

L.F. Dell'Osso, Ph.D., Professor Emeritus of Neurology Daroff-Dell'Osso Ocular Motility Laboratory Louis Stokes Cleveland Veterans Affairs Medical Center 10701 East Boulevard, Cleveland, OH 44106 USA Telephone: (216) 421-3224-FAX: (216) 231-3461 E-mail:1fd@case.edu-Web Site: www.omlab.org forms, where the jerk slow phases are usually increasing velocity (or "runaway") exponentials. These accelerating slow phases are always towards the neutral zone (Dell'Osso \& Daroff, 1975; Dell'Osso, Flynn, \& Daroff, 1974). The primary subsystem instability in INS is hypothesized to lie in the normally underdamped smooth pursuit system; vestibular dysfunction (imbalance) may also be present and would account for the less-prevalent linear waveforms seen in some patients. In individuals demonstrating an additional high-frequency sinusoidal oscillation (producing dual jerk waveforms), the 
nucleus of the optic tract may be involved (Dell'Osso \& Daroff, 1981).

The original ocular motor system (OMS) model (and the subsequent versions published in our studies) is a top-down, control systems model reproducing the ocular motor responses of normal individuals as well as those with several INS waveforms (Jacobs \& Dell'Osso, 2004). This behavioral OMS model evolved from earlier models that were based on years of observation and analysis of normal and abnormal eyemovement data (Dell'Osso, 2002b); wherever applicable, adherence to known anatomical structure was maintained. Emphasis is put less on where each functional block is located and more on and how they work together (i.e., the communication between them) under organizing principles resulting in known ocular motor system behaviors. (These publications contain block diagrams of prior versions that may be compared with Figures 1 and 9). Figure 1 is a block diagram of the current model outlining the important functional blocks and their interconnections.

OMS Block Diagram

(v1.5)

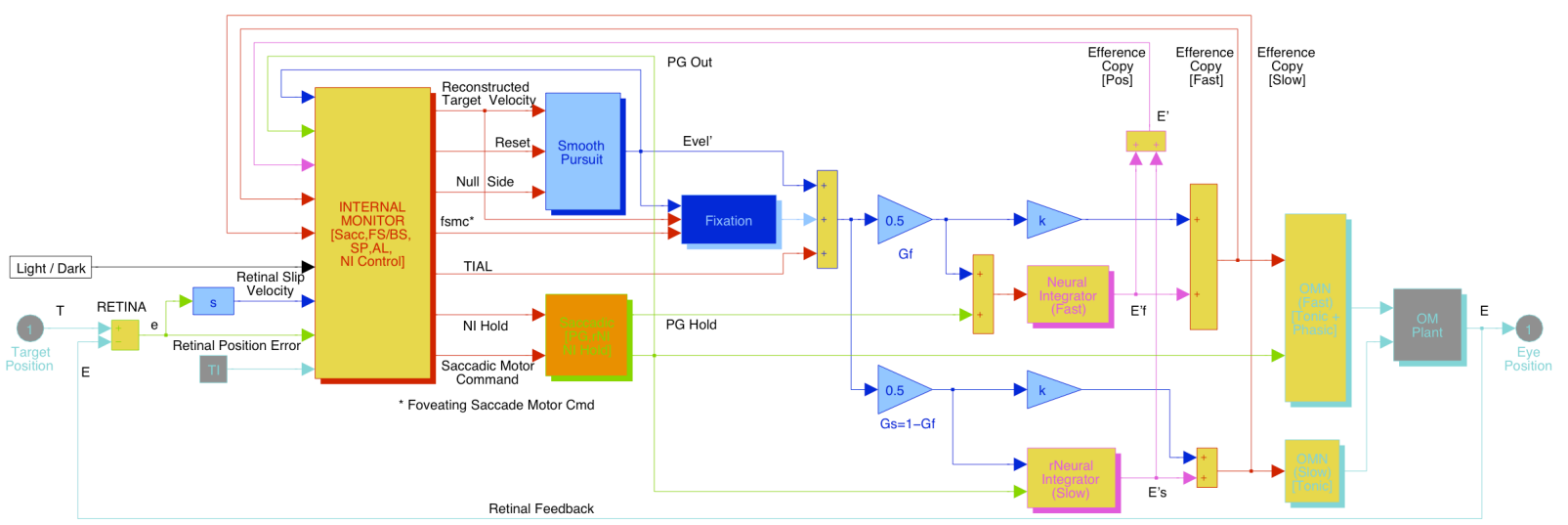

Figure 1. Block diagram of the Ocular Motor System model (version 1.5). The changes made in the current version were within the Internal Monitor, Smooth Pursuit, and Neural Integrator functional blocks and some of their interconnections.

There are, however, a number of important INS characteristics that were not included in our original OMS model. To expand that behavioral model, we first incorporated the gaze-angle variation commonly observed in most INS patients in an interim version of the model. Alexander's law (Doslak, Dell'Osso, \& Daroff, 1982; Robinson et al., 1984) describes the increase in the amplitude of nystagmus as the eye is moved in the direction of the fast phase in Vestibular Nystagmus (VN) and Fusion Maldevelopment Nystagmus Syndrome (FMNS). The slope of the Alexander's law relationship is dependent on the tonic imbalance (in $\mathrm{VN}$ ) or the viewing eye (in FMNS). Although most INS slow-phase waveforms exhibit different accelerations than $\mathrm{VN}$ and FMNS waveforms, we hypothesized that the same Alexander's Law output was responsible for the amplitude changes affecting the eXpanded Nystagmus Acuity Function (NAFX) peak, i.e., the INS "null” (Dell'Osso \& Jacobs, 2002). 
After successfully implementing the gazeangle variations in the model, we demonstrated that a unifying mechanism was capable of producing the various pendular and jerk waveforms of INS. A novel neural integrator in the pursuit pre-motor circuitry $(\mathrm{PMC}+)$ was designed to reset the underlying pendular oscillation when each foveating fast phase is made. The Alexander's law variation also governed the simulation of the idiosyncratic transitions from one type of waveform to the other and their changes with gaze angle.

\section{Methods}

\section{Recording}

The ocular motor recordings and observations used for the computer simulations came from approximately 1000 subjects with INS (ranging from newborn to the elderly), who were recorded in our laboratory over the past 40 years. Written assent and consent was obtained from subjects before the testing in accordance with our institutional IRB. Subjects were seated in a chair with headrest or a chin stabilizer, far enough from an arc of red LEDs, or a reflected laser spot, to prevent convergence effects $(>5$ feet). At this distance the non-stress-inducing target subtended less than $0.1^{\circ}$ of visual angle. The room light could be adjusted from dim to blackout to minimize extraneous visual stimuli. Experiments usually consisted of from eight to ten trials, each lasting a few minutes with time allowed between trials for the subject to rest. Trials were kept this short to guard against boredom because INS intensity decrease and waveform deterioration are known to occur with inattention (this is illustrated below in Figure 6).

Eye movements were measured using an infrared reflection (IR, eye-trac 210, ASL, Waltham,
MA), a magnetic scleral search coil (C-N-C Engineering, Seattle, WA), or a high-speed digital video (EyeLink II, SR Research, Mississauga, ON, Canada) system. The IR system was linear to $\pm 20^{\circ}$ in the horizontal plane and monotonic to $25-30^{\circ}$ with a sensitivity of $0.25^{\circ}$. The search-coil system had a linear range greater than $\pm 20^{\circ}$, a sensitivity of $0.1^{\circ}$, and crosstalk less than $2.5 \%$. Each coil was pre-calibrated using a protractor device. The digital video system had a linear range of $\pm 30^{\circ}$ horizontally and $\pm 20^{\circ}$ vertically. System sampling frequency was $500 \mathrm{~Hz}$; gaze position accuracy error was $0.5^{\circ}$ $1^{\circ}$ on average. The total system bandwidth for all systems (position and velocity) was $0-100$ $\mathrm{Hz}$. The data from all systems were digitized at $500 \mathrm{~Hz}$ with 16-bit resolution.

The position signal for each eye was calibrated with the other eye behind cover to obtain accurate position information for both; the foveation periods were used for zero-adjustment and calibration. Eye positions and velocities (obtained by analog differentiation of the position channels) were displayed on a strip-chart recording system. Monocular primary-position adjustments for all methods allowed accurate position information and documentation of small tropias and phorias hidden by the nystagmus.

\section{Analysis and Simulation}

All the analysis and graphics were done in the MATLAB environment (The MathWorks, Natick, MA) using the OMtools software available on http://www.omlab.org ("Software and OMS Models" page). Only eye position was sampled directly; velocity was derived from the position data by a 4th order central-point differentiator; acceleration was derived from the velocity data by the same differentiator. Position data were pre-filtered with a low-pass filter with the cutoff frequency of $50 \mathrm{~Hz}$ to eliminate the noise without changing the nystagmus signals to be studied. Only data from the fixating eyes 
were analyzed. All ocular motor simulations were performed using MATLAB Simulink (Waltham, MA).

\section{Results}

\section{Gaze-Angle Variation}

As in the original OMS model, the Alexander's law mechanism uses efference copy of eye position to modulate the tonic imbalance (TI) input (Dell'Osso \& Jacobs, 2001; Jacobs, 2001; Jacobs \& Dell'Osso, 2004). The eye-position signal is multiplied by the Alexander's law slope and filtered before summing with TI. The sign of TI dictates if this sum is kept greater than or less than 0 . Then the sum is passed to a final switch that only produces an output when TI is present. Varying the Alexander's Law slope produces differing amounts of Alexander's Law effect. This effect leads to slowphase velocity increases as gaze is directed in the abducting direction of the fixating eye, and that would cause the transition from foveating to defoveating fast phases in FMNS (Dell'Osso \& Jacobs, 2001). In both INS and FMNS, the foveating saccades are also referred to as fast phases or quick phases.

For FMNS patients, the two Alexander's law slopes (one for each eye) operate independently of each other, with only one (depending on the fixating eye) determining the gaze-angle variation (Dell'Osso \& Jacobs, 2001). In INS, we hypothesize that both Alexander's law relationships operate together-it is the same Alexander's-law imbalance produced by improper calibration of the vestibular system that may be the underlying reason for INS gaze-angle variation. The two linear functions act simultaneously, with their intersection establishing the null position and the slopes of the lines controlling the broadness of the null. The modulation is produced by a variable gain in the $\mathrm{PMC}+$ block (Jacobs \& Dell'Osso, 2004; Robinson, Gordon, \& Gordon, 1986), controlling the amplitude of the pendular pursuit-velocity oscillation.

In the modified Alexander's law block, efference copy of the eye position is used as an input to calculate the modulation factor (the "Null Mod" output). The efference copy of eye position used here is an internally generated, nystagmus-free position signal, i.e., the desired eye position; it provides a stable signal for the oscillation amplitude modulation. In the absence of a TI, the difference between the current eye position and the null dictates the oscillation amplitude. Future versions of the model that could also simulate INS with a latent component will require the addition of a fixating eye signal to this block. Figure 2 shows the initial changes to the $\mathrm{PMC}+$ circuit, the origin of the nystagmus oscillation. The first multiplier in the feedforward loop is where the modulation from Alexander's law takes place. The second multiplier is used to simulate the effects of centrally acting drug therapy on INS (e.g., the value shown as 0.6 ); its normal value (no drug therapy) is 1.0 .

Figures 3 and 4 demonstrate model position outputs for different gaze-angle variations, simulating different null positions and widths. Note that the voluntary saccades are still accurate and foveating saccades are also accurate, once target acquisition occurs. However, the farther away from the null position, the less "flat" the foveation periods. This is due to the fixation system's response to a higher velocity input (Dell'Osso, 2002a). Now that eye position guides the oscillation amplitude, gaze-holding ability also varies with different eye positions. This emergent property is consistent with eyemovement data of INS patients. 
Figure 5 shows the model outputs to a series of step stimuli for two different null positions. The eye position output simulates the reduced oscillation within the null zone and enlarged oscillation lateral to the null zone; the extent of the enlargement depends on the null width (in both simulations, the null width is set to "medium", similar to Figure 4). These robust model outputs duplicate recordings of patients with pendular types of waveforms under step visual stimuli (i.e., stationary targets that jump to different positions).

\section{The Unifying Hypothesis}

Historically, several hypotheses have been suggested for the origin of jerk types of INS waveforms. In this section, they will be discussed using human ocular motor data in support or contradiction of them. This process was used to evolve the original OMS model hypothesis to generate jerk waveforms and behaviors and was closely correlated with the data recordings accumulated in our lab over the past four decades.

The first hypothesis was that the accelerating IN slow phases were caused by excessive positive feedback around the normally leaky $(\tau=25 \mathrm{sec})$ neural integrator (NI). In control-systems analysis, this corresponds to a right-half plane pole (Dell'Osso \& Daroff, 1981). However, INS patients with jerk waveforms who simultaneously had gaze-holding problems, have been described (Dell'Osso, Weissman, Leigh, Abel, \& Sheth, 1993). In patients with a combination of INS plus gaze-holding failure, both eyes drift back after a voluntary saccade while there is an ongoing INS waveform with accelerating slow phases. The eyes' drifting is a clear indication of insufficient gain in the NI feedback loop; the fact that it can co-exist with the accelerating jerk waveforms contradicts the hypothesis of excessive gain in that loop (for the same person, a gain can not be both insufficient and excessive).

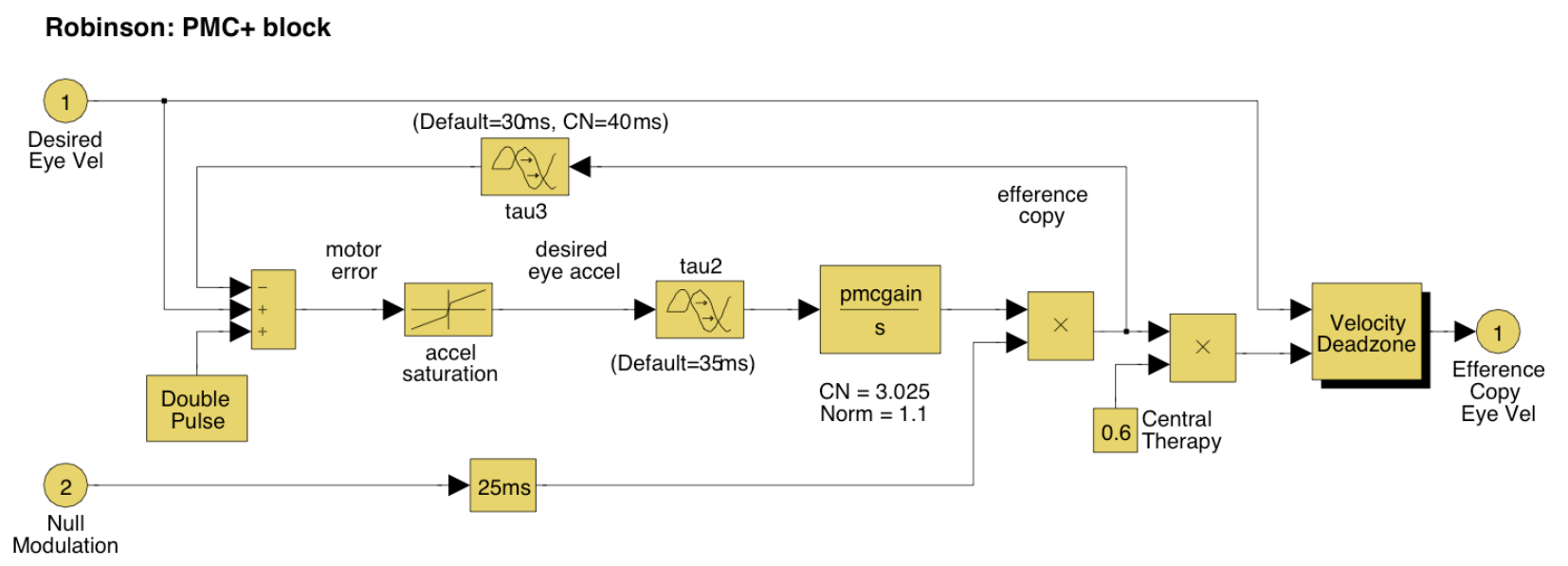

Figure 2. The initial changes to the PMC+ circuit in the modified Ocular Motor System model to allow Alexander's law modulation of the INS amplitude via the new input, "Null Modulation" that is applied through the first multiplier. The new "Velocity Deadzone" block is used to exclude artifacts in the simulations for normals, i.e., the initial underdamping of the pursuit system. Also, the new "Central Therapy" is applied through a second multiplier to simulate the damping effects of drug therapy (default value for no therapy is $1.0)$. 


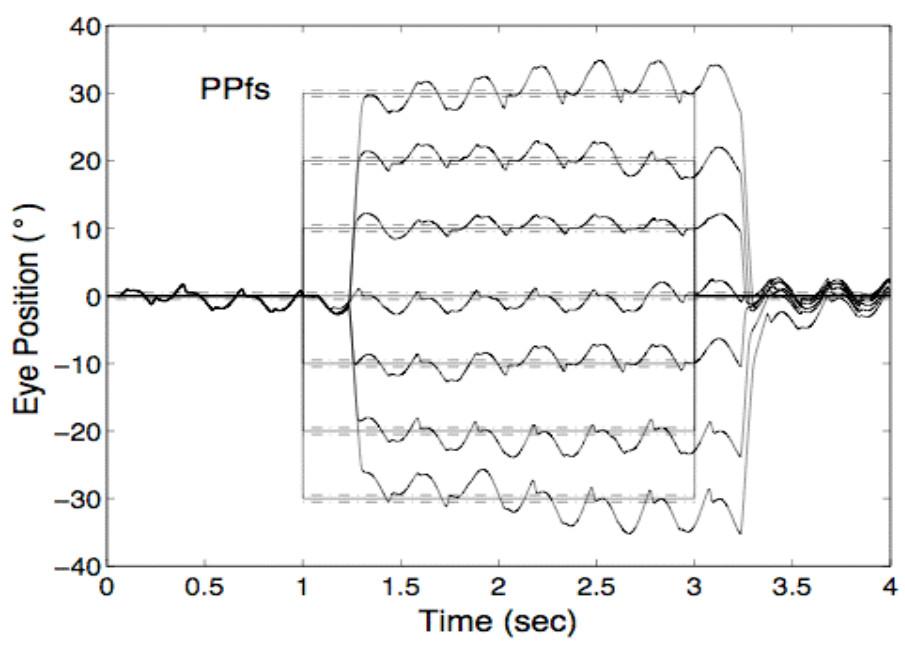

Sharp AL Intersection at $10^{\circ}$
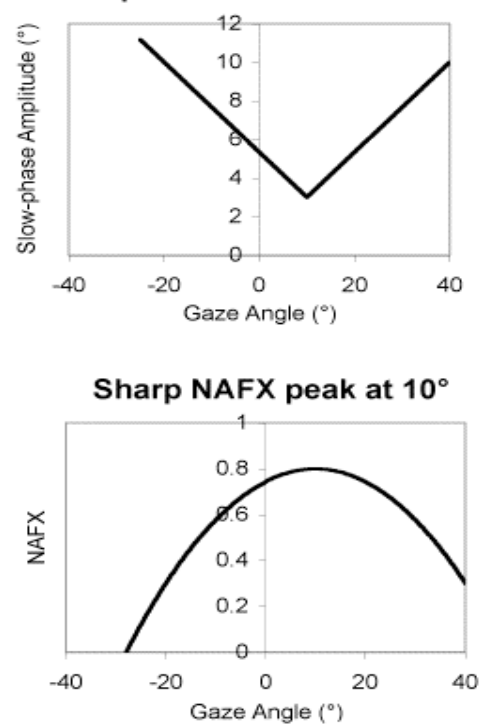

Figure 3. Model simulations of PPfs INS waveform with a sharp null at $10^{\circ}$ and increased slow-phase amplitude to either side of the null. Note the accuracy of both gaze shifting and holding at all gaze angles despite the INS. In this Figure and Figure 4, right columns show the intersection and slopes of the two Alexander's Law lines, and the peak position and sharpness of the eXpanded Nystagmus Acuity Function (NAFX) vs. gaze-angle curve, corresponding to the position and sharpness of the null. The dot-dashed lines are an indication of the $\pm 0.5^{\circ}$ fovea.
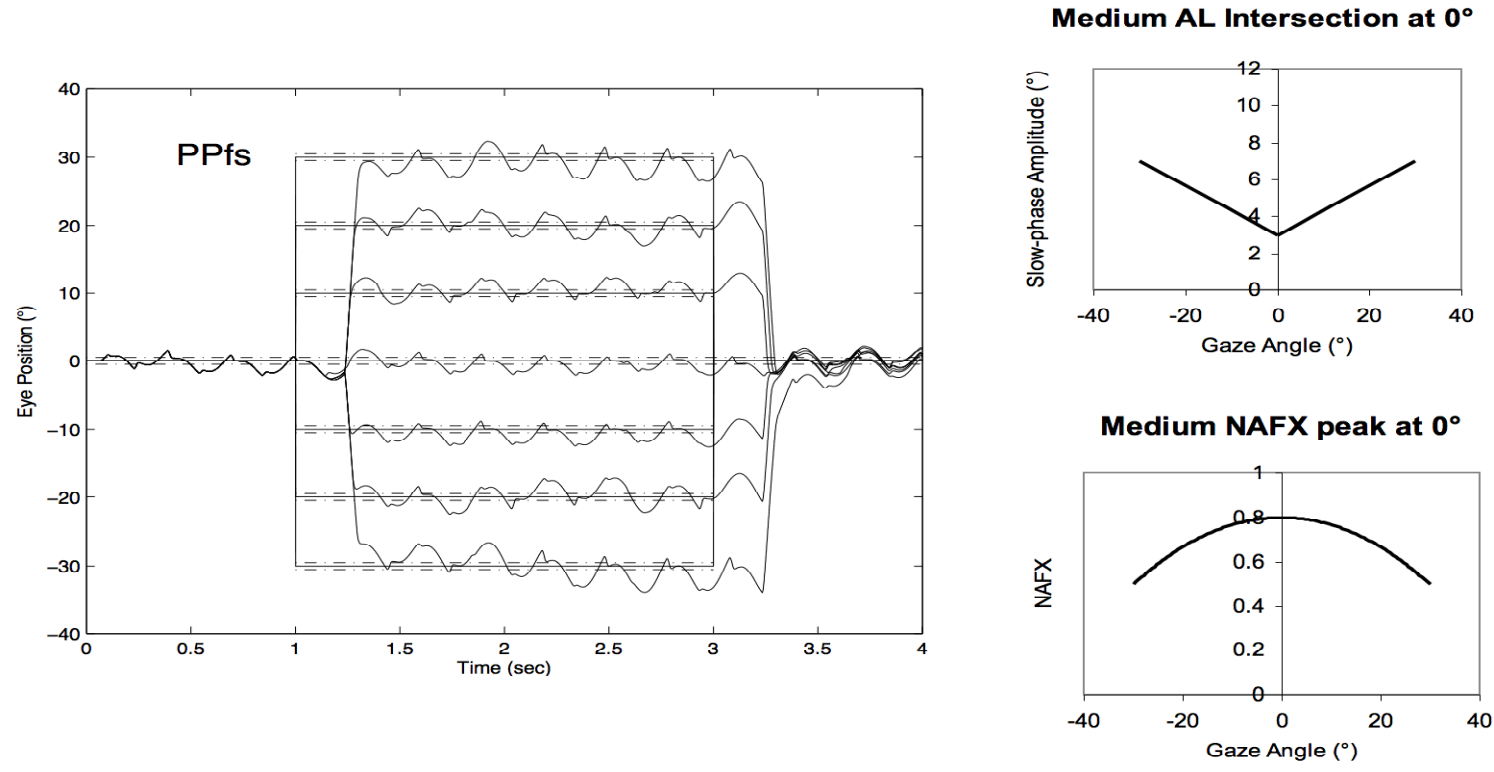

Figure 4. Model simulations of PPfs INS waveform with a medium null at $0^{\circ}$ and increased slow-phase amplitude to either side of the null. Note the accurate target foveations at all gaze angles despite differences in INS amplitudes and occasional bias shifts. The dot-dashed lines are an indication of the $\pm 0.5^{\circ}$ fovea. 

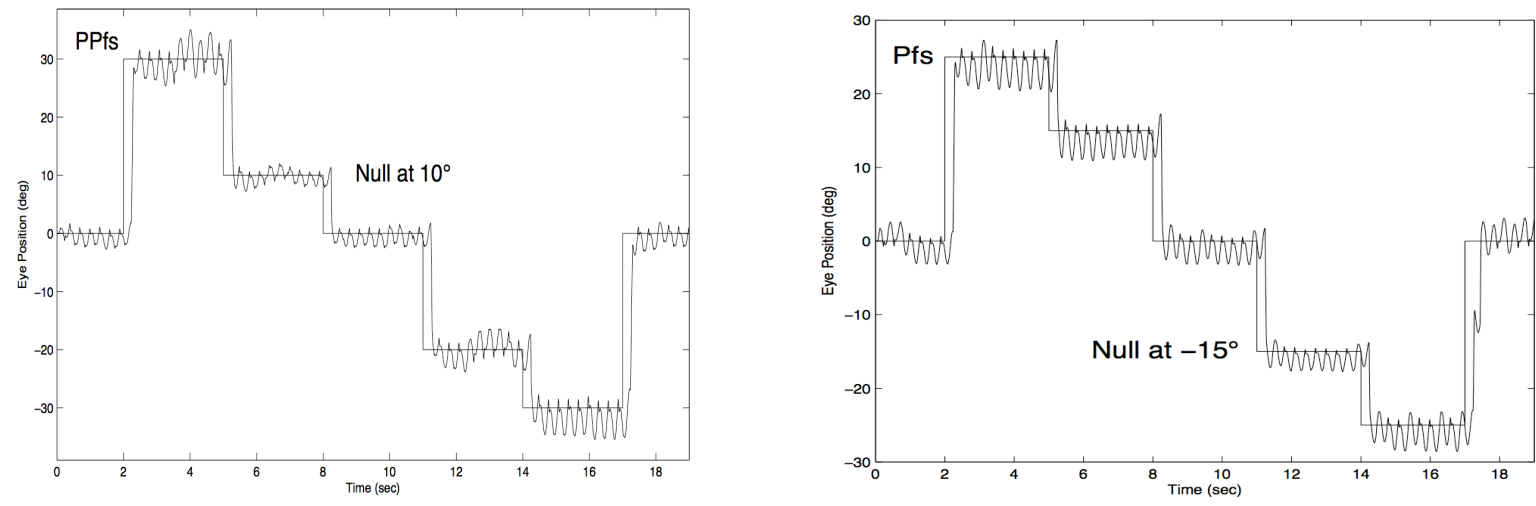

Figure 5. Model simulations of PPfs step responses of INS with a medium null at $10^{\circ}$ (left) and $-15^{\circ}$ (right). Note the accurate target foveations at all gaze angles despite differences in INS amplitudes and occasional bias shifts.

Another fact that disproves the excessive-gain hypothesis is that it would result in centrifugal acceleration but IN slow phases accelerate centripetally to the neutral zone. Therefore, although it is possible to generate jerk waveforms with an excessive-gain around the NI, these waveforms only superficially resemble IN, i.e., they are not behavioral and the putative mechanism is a not realistic model of INS.

A second hypothesis for jerk waveforms claimed they originated from the saccadic system. However, several aspects of INS behavior strongly suggest that the saccadic system is normal in INS and unrelated to the genesis of the oscillation (Dell'Osso, 2006):

1. For static fixation, IN intrinsic saccades are always corrective in their direction, and two types accurately foveate the target (foveating saccades and foveating fast phases);

2. Spontaneous changes in slow-phase direction/acceleration occur in the $a b$ sence of saccades;

3. After IN damping (e.g., due to inattention), new slow phases begin without a preceding saccade;
4. Gaze-angle variation of IN slow phases (amplitude or direction) is independent of the presence or amplitudes of saccades;

5. Voluntary saccades do not alter the amplitude or direction of IN slow phases and lead to accurate target foveation.

The intrinsic saccades in the INS cycle are adaptations of the OMS for target foveation and the initiation and modulation of the INS oscillations are independent of both intrinsic and voluntary saccades. If anything, the saccadic system is performing extraordinarily well under the constantly present pendular oscillation; i cannot be the cause of that oscillation. Thelefore, simple waveform models that are based on the saccadic hypothesis also produce waveforms that only superficially resemble those of INS and are neither realistic nor capable of simulating known INS behavior.

Re-examination of INS inattention data found in the recordings of many subjects, suggested that the oscillation originated in the pursuit system. In this paper and the model, the foveating saccades made in the jerk waveforms, J and Jef, 
are termed "foveating fast phases" to differentiate them from the foveating saccades in pendular with foveating saccades (Pfs) and pseudopendular with foveating saccades (PPfs) waveforms. In some patients with alternating direction jerk INS waveforms whose slow phases revert to pendular during inattention, the fast phases are suppressed during the inattention and the underlying pendular component is revealed. In others, jerk INS degenerates to pendular INS with inattention; the jerk waveform returns immediately upon verbal prompting, inhibiting the larger pendular component. In both cases, the eyes move farther from the target. Figure 6 demonstrates some examples of enlarged show phases (including points of inflection) due to gradually occurring inattention. In this Figure, the foveating fast phase was delayed and the accelerating slow phase actually decelerated before the fast phase reset the fovea on target. As soon as attention to the target was reestablished (either spontaneously or after ver-

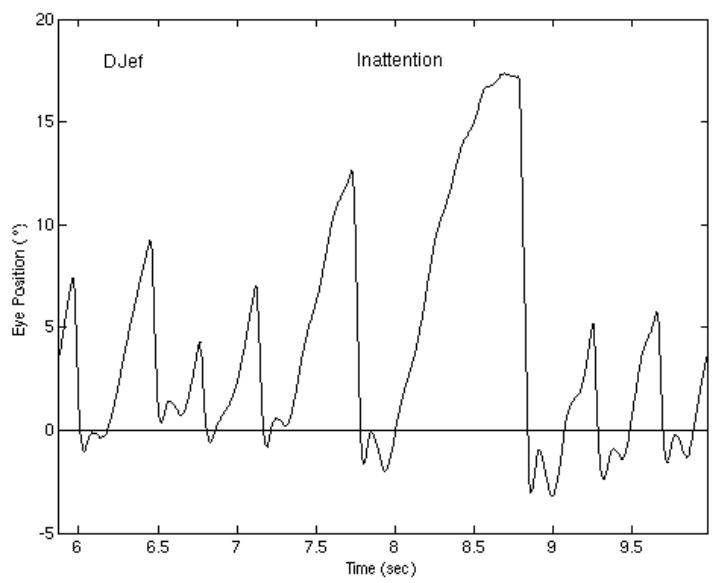

bal prompting by the experimenter), a foveating fast phase was generated and jerk waveforms reoccurred. These effects can occur repeatedly, especially in young patients, as shown in the right panel of Figure 6.

Therefore, for static fixation, inattention: 1) suppresses corrective saccades; 2) results in a larger IN; and 3) uncovers the underlying pendular oscillation for most INS waveforms. Both the pendular and jerk waveforms are essentially pendular, as a result of poor calibration of the evolved damped instability in the smooth pursuit system. This unifying hypothesis was then incorporated into our OMS model. Most other models of INS are simply "waveform generators" consisting of sub-portions of the OMS and are incapable of simulating behavioral responses, accurate, prolonged foveation periods, or the changing waveform characteristics of INS.

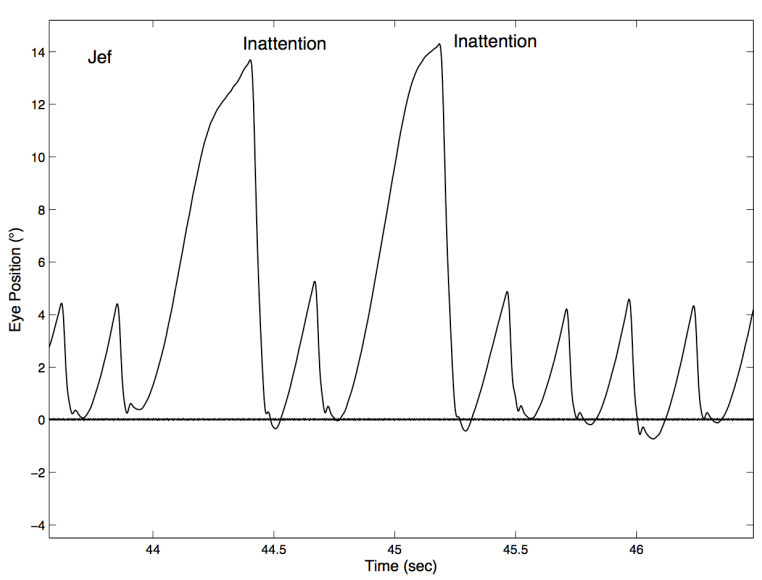

Figure 6. Human data showing fixation in primary position with duel jerk waveforms whose slow phases become enlarged as inattention gradually occurs (left); Fixation in primary position showing the waxing and waning of attention (right). DJef = Dual Jerk with Extended Foveation; Jef = Jerk with Extended Foveation.

\section{Jerk Waveform Generation}

We used the same "evolutionary" procedure to generate jerk waveforms as had been employed previously for pendular waveforms
(Jacobs \& Dell'Osso, 2004). To change the system's underlying pendular oscillation, the saccadic subsystem required modification to correctly perform under those conditions when foveating fast phases should be gener- 
ated. Table 1 contains comparisons among the intrinsic saccades made in both pendular and jerk waveforms. The transition from a PPfs waveform to a jerk waveform requires suppression of braking saccades and their replacement with fast phases. To accomplish this, we first altered the functional block ("Breaking/Foveating Saccade Logic") within the Internal Monitor. This block contains the logic that would normally trigger the braking saccades of IN or fast phases of induced nystagmus (e.g., vestibular or optokinetic). The first, simple modification to that functional block gave rise to alternating direction jerk nystagmus (i.e., unidirectional jerk cycles whose foveating fast phases were made in alternating directions from both sides of the target) (Wang, 2008).
To convert an alternating direction jerk waveform into a unidirectional jerk waveform, additional modifications were necessary. Firstly, the underlying pendular oscillation needed to be reset when each foveating fast phase was made. The use of a resettable neural integrator in $\mathrm{PMC}+$ accomplished the resetting (Figure 7, top). This neural integrator has the same structure as the one in pulse generator (Abel, Dell'Osso, \& Daroff, 1978; Abel, Dell'Osso, Schmidt, \& Daroff, 1980; Kustov \& Robinson, 1995), distinct from the common neural integrator that appears in the final motor pathway. We used the motor command for foveating fast phases as a resetting signal, i.e., when the BS/FS logic box indicated the need to generate a foveating fast phase, the pendular oscillation underlying pendular INS waveforms was reset.

\begin{tabular}{|l|l|l|l|}
\hline \multirow{2}{*}{} & \multicolumn{2}{|c|}{ PPfs } & \multicolumn{1}{|c|}{ Jerk } \\
\cline { 2 - 4 } & Braking Saccade & Foveating Saccade & Foveating Fast Phase \\
\hline $\begin{array}{l}\text { Position } \\
\text { Criteria }\end{array}$ & $\begin{array}{l}\text { Eye running away } \\
\text { from the target }\end{array}$ & $\begin{array}{l}\text { Eye approaching the } \\
\text { target }\end{array}$ & $\begin{array}{l}\text { Eye running away from } \\
\text { the target }\end{array}$ \\
\hline $\begin{array}{l}\text { Amplitude } \\
\text { and } \\
\text { Direction } \\
\text { Calculation }\end{array}$ & $\begin{array}{l}\text { Stereotyped (fixed } \\
\text { amplitude) }\end{array}$ & $\begin{array}{l}\text { Should accurately predict } \\
\text { where the eye will be 60 } \\
\text { ms later (default value } \\
\text { based on distribution of } \\
\text { internal delays) }\end{array}$ & $\begin{array}{l}\text { Should accurately predict } \\
\text { where the eye will be 60 } \\
\text { ms later on default value } \\
\text { based intibution of } \\
\text { internal delays) }\end{array}$ \\
\hline $\begin{array}{l}\text { Velocity } \\
\text { Criteria }\end{array}$ & Velocity exceeds user-settable thresholds for idiosyncratic waveforms \\
\hline $\begin{array}{l}\text { Acceleration } \\
\text { Criteria }\end{array}$ & Acceleration is below user-settable thresholds for idiosyncratic waveforms \\
\hline
\end{tabular}

Table 1. Comparison of braking and foveating saccades in PPfs and foveating fast phases in jerk waveforms.

The foveating fast-phase motor command was appropriately prolonged (35 $\mathrm{ms})$ and delayed ( 5 ms). Due to the time delays in both the feedback and feedforward loops, the resetting re- 
quired that those time delays also be reset (i.e., the stored energy needed to be dumped). The "Null Mod" block in the PMC+ (Figure 7, bottom) is used to determine the starting direction of the oscillation, which in turn dictates the fastphase direction.

Secondly, the outputs of the Alexander's law circuit were rearranged. A "Null Side" output was used to determine which side of (and how far from) the neutral zone the current eye position is, based on efference-copy estimation. The neutral zone is defined as the gaze angles where pendular type waveforms exist; for some patients, it can be different than the null zone (the gaze angles where the amplitude/intensity is lowest). Most of the time, neutral zone is referred to when waveform transition is discussed; the null zone (more accurately, the NAFX peak) is referred to in regard to visual function. The "Null Side" Alexander's law output enables the transitions among waveforms and allows the simulated slow phases to accelerate centripetally toward the neutral zone. Figure 8 shows the Alexander's law circuitry used in this model, which is a slightly modified version of the prior version; for simplicity, part of the latter was relocated as the "Null Mod" block within the PMC+ block.

Thirdly, the BS/FS logic was further modified to accurately generate foveating fast phases in jerk waveforms. Those fast phases differ from the foveating and braking saccades in the PPfs waveforms (see Table). Braking saccades are automatically generated to brake runaway eye velocities. For the PPfs waveform, the logic necessary to decide whether a saccade will be braking or foveating is the following: If the eye is running away from the target at the time of saccade programming (which precedes the actual time the saccade is generated), the velocity

PMC+ block

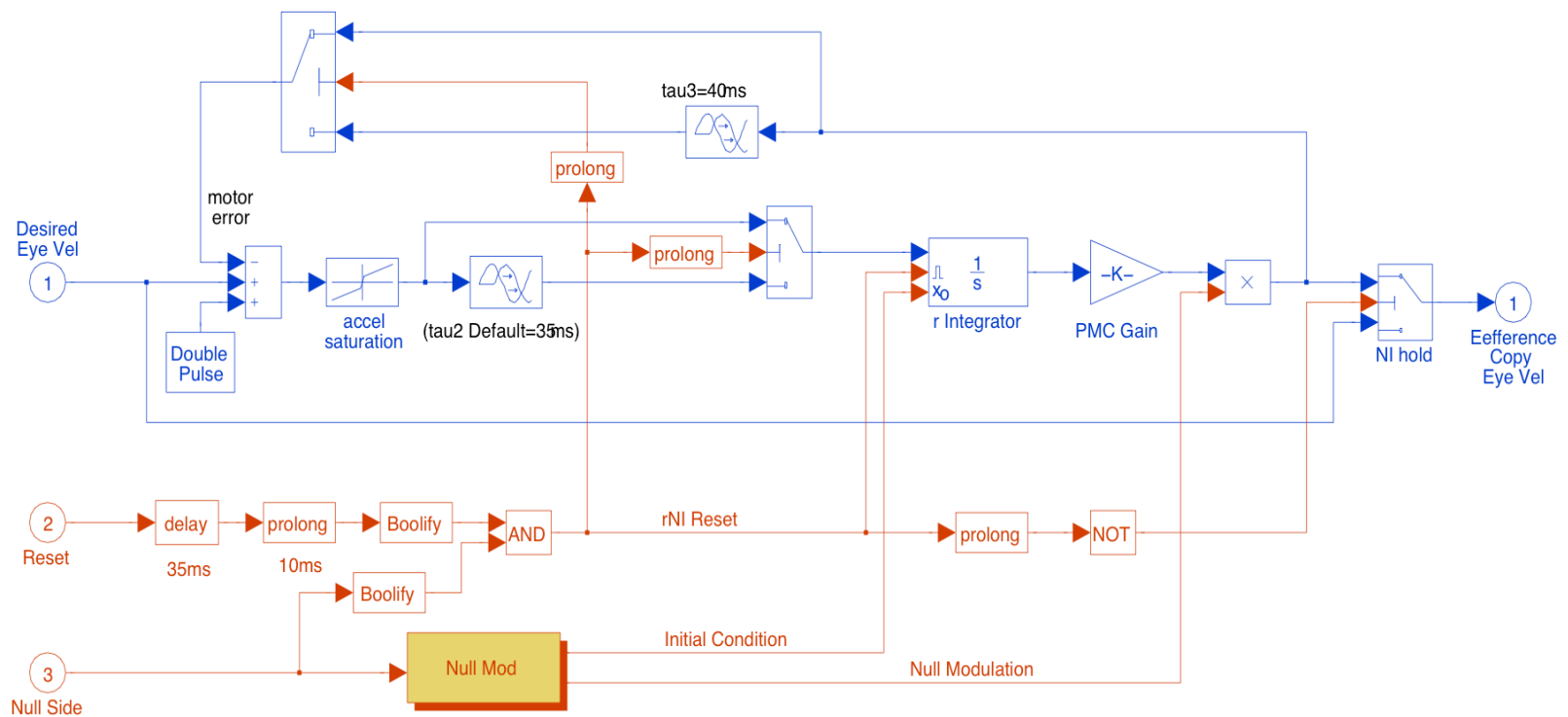




\section{Null Modulation}

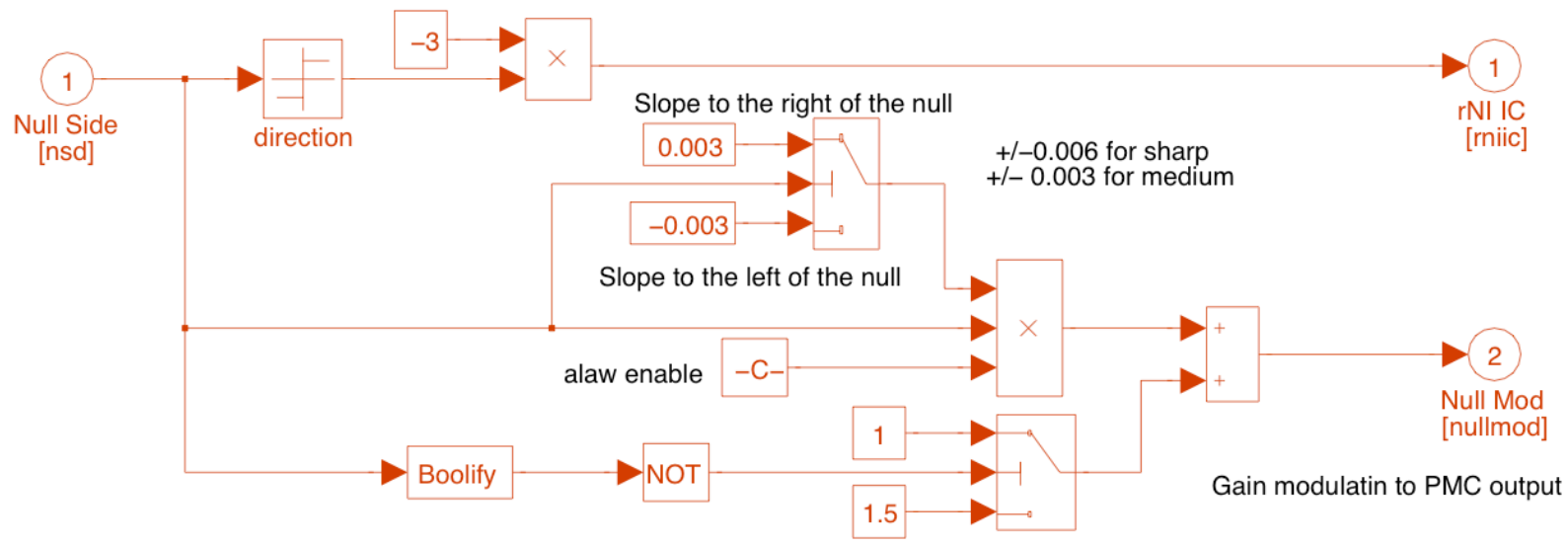

Figure 7. Modified premotor circuitry (PMC+) block (top) in the OMS model. This modified PMC + block now generates a restarted oscillation for every jerk nystagmus cycle, using new resetting signals (delayed, prolonged and boolified) from the foveating fast phase motor command (fpmc). The output ("Efference Copy Eye Vel') now resets to 'Desired Eye Vel." The output of the relocated "Null Mod" block is used to modulate the INS oscillation via the final multiplier. Expansion of "Null Mod" block (bottom) demonstrating how the null sharpness may be set and the initial condition to the resettable integrator is generated.

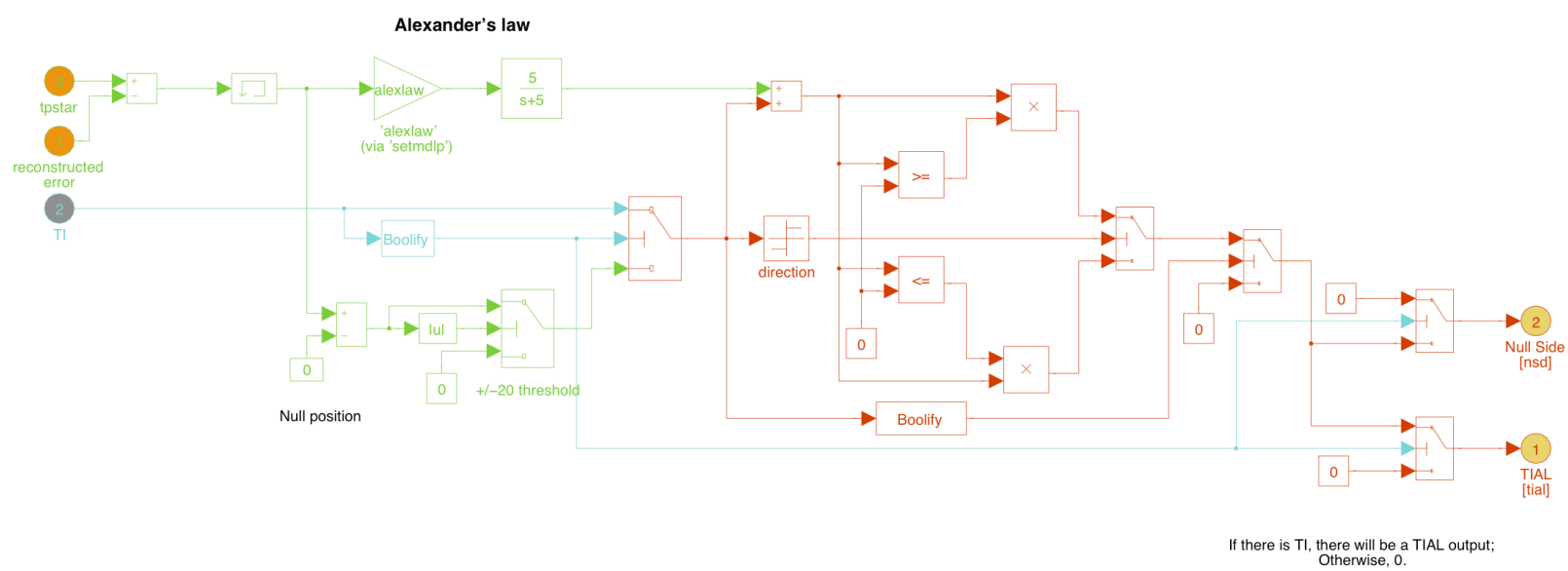

Figure 8. Modified Alexander's law block generating the new "Null Side" signal that is used by the PMC+ block (shown in Figure 7) in addition to the original "TIAL" output.

exceeds a user-settable threshold (default = $4 \%$ ), and has passed its point of maximum velocity (i.e., is not still accelerating), a braking saccade will be generated. This is consistent with the definition of a braking saccade (Dell'Osso \& Daroff, 1976; Jacobs, Dell'Osso,
\& Erchul, 1999). If, however, the eye is approaching the target at that time and the velocity exceeds the threshold (and falls below the acceleration threshold), then the saccade will be foveating. Its magnitude and direction will be calculated by the predicting where the eye will 
be $60 \mathrm{~ms}$ later (default value based on the current distribution of internal delays in the model), when the saccade will actually occur. In jerk waveforms, foveating fast phases are made when the eye is running away from target, the same as braking saccades. Under this circumstance, the logic for fast-phase timing was the same as for braking saccades in the PPfs case. However, there are distinct differences between the amplitudes of those two types of saccades. Braking saccades have small, stereotyped amplitudes independent of the eye's position vis-àvis the target, while the foveating fast phases of jerk waveforms are larger and must accurately foveate the target by correcting the position error. The required magnitude is calculated by predicting where the eye would be $60 \mathrm{~ms}$ later (when the saccade would occur), similar to the method for calculating the amplitudes of foveating saccades in PPfs.

The last modification required was to the neural integrator for the group of slow motor neurons.

OMS Model (v1.5)
We incorporated the jerk waveform changes into our model containing separate groups of motor neurons for the slow and fast eye movements (Dell'Osso \& Wang, 2008; Ugolini et al., 2006; Wang, Dell'Osso, Zhang, Leigh, \& Jacobs, 2006; Wang, 2008). A potential

problem occurs with such a model when the pursuit-system instability generates a velocity signal with a DC value (as would be the case for unidirectional jerk waveforms). The integrated output of the slow NI (the NI neurons associated with the slow motor neuron pathway) would constantly grow due to that DC value, whereas the output of the fast NI would be reset by its saccadic input. Left unchecked, the slow NI output could grow unbounded as the simulation ran, although the summed outputs of the fast and slow NIs would still reflect the true current eye position. Because the NI represents a group of firing neurons, a potentially infinite output is not physiological. In the pendular waveforms we simulated using the original model (Jacobs \& Dell'Osso, 2004), the

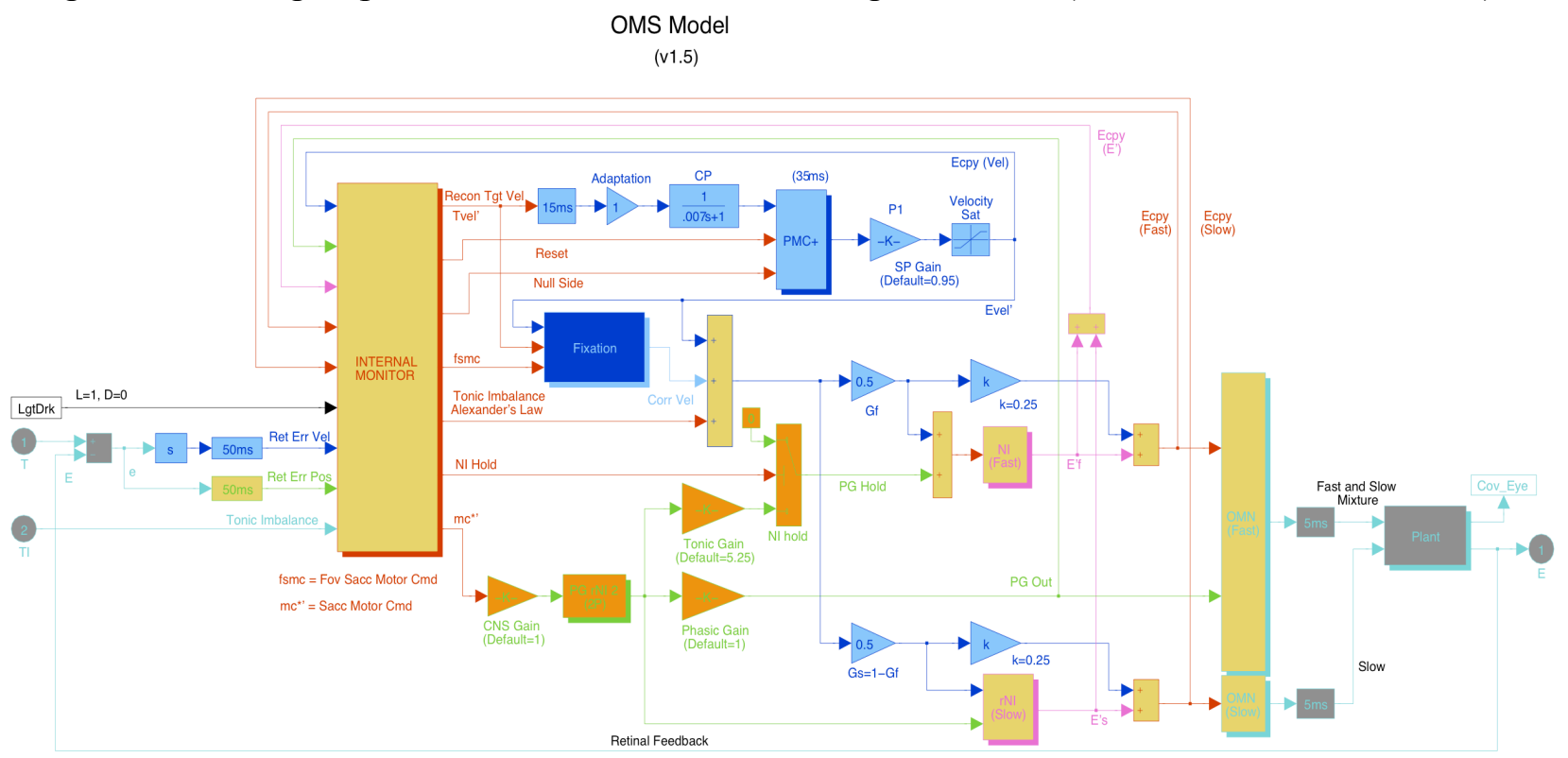

Figure 9. Updated OMS model that also simulates jerk INS waveforms. Compared to earlier versions of the model, changes were made within the INTERNAL MONITOR (Alexander's Law), the PMC+ blocks, and the signals to the PMC+ block. Also, the two final common integrators "NI (Fast)" and "NI (Slow)" now simulate their respective neural populations. 

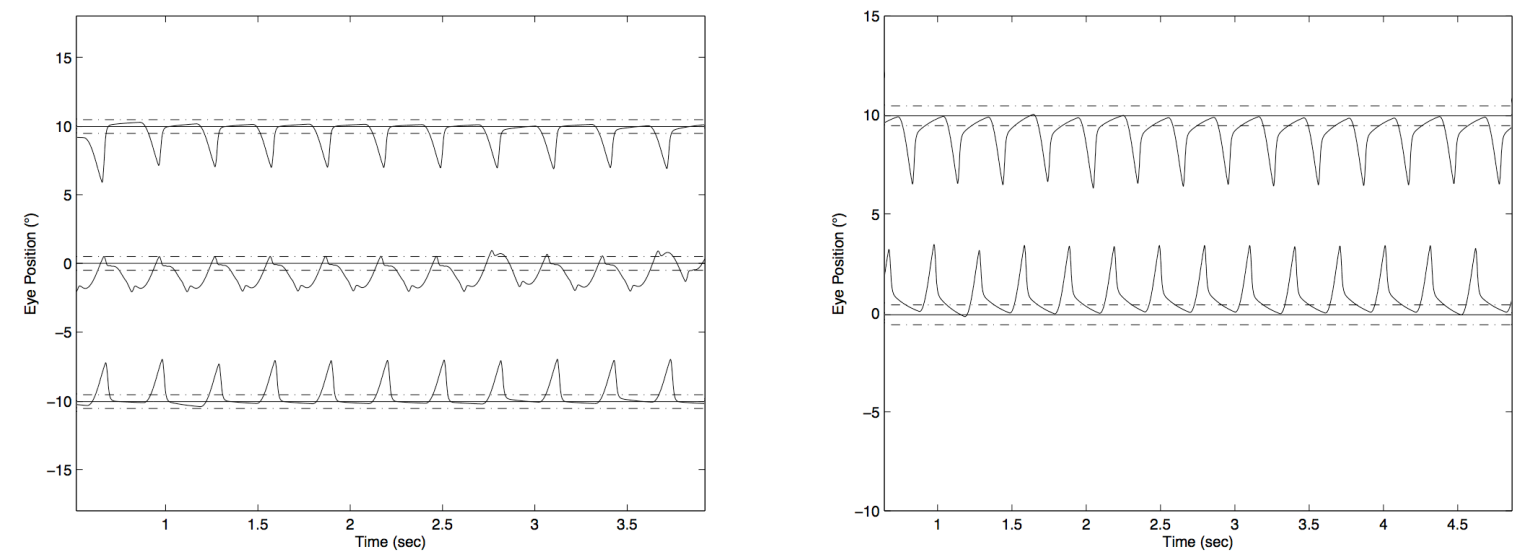

Figure 10. (left) OMS model simulations of $0^{\circ}$ fixation comparing jerk and pendular waveforms; jerk right and jerk left waveforms are plotted at different locations $\left(10^{\circ}\right.$ and $-10^{\circ}$, respectively) for clarity. (right) OMS model simulations of $0^{\circ}$ fixation during pseudocycloid waveforms in either direction; right pseudocycloid waveforms are plotted at a different location $\left(10^{\circ}\right)$ for clarity. Note the accurate target foveations despite differences in INS waveforms and the longer foveation periods common in jerk with extended foveation waveforms. The dot-dashed lines are an indication of the $\pm 0.5^{\circ}$ fovea.

DC value of the sine-wave velocity oscillation was always 0 , therefore this problem did not arise when the single NI was expanded to simulate both the fast and slow NI populations.

We hypothesize that although the slow NI does not integrate the saccadic pulse signals, it could use signals derived from saccadic pulses to regulate its output. When the input has a DC value, the saccadic signals reset the slow NI output, similar to what saccadic pulses do automatically to the fast NI. The Model shown in Figure 9 has been updated with a resettable slow NI that eliminates the problems caused by oscillations with non-zero DC values.

The modifications described above allowed accurate and behaviorally correct simulations of unidirectional jerk waveforms. Some fixation simulations are displayed in Figure 10 (left). The three traces are all fixation at $0^{\circ}$; jerk-right and jerk-left waveforms are plotted at different locations for clarity. The PPfs simulation is shown for comparison. The dot-dashed lines are indication of the $\pm 0.5^{\circ}$ fovea. In all three wave- forms, although there were slight differences between the sizes of each saccade (as commonly seen in human data), the final targetimage position always remained within the \pm $0.5^{\circ}$ foveal area (which allows the best visual acuity). Like most pendular waveforms recorded in humans, the foveation periods generated by the model extend up to $50 \mathrm{~ms}$. The jerk waveforms simulated here have a much longer and flatter foveation period right on target (up to $300 \mathrm{~ms}$ ), suggesting good visual function. An interesting emergent property is that if we reduce the calculation accuracy for the fast phases in jerk waveform generation (a scale factor in the model), pseudocycloid waveforms result, as shown in Figure 10 (right). The pseudocycloid waveform is common in INS and, in our model, requires no additional functional ocular motor blocks.

The model also accurately simulated responses to step changes in target position and smoothly transitioned to another type of waveform depending on eye position (Figure 11). In this Figure, the neutral zone of the "simulated sub- 
ject" is set to be $\pm 5^{\circ}$ around the primaryposition null; as long as the intended eye position is in this range, pendular waveforms will result. Right lateral gaze beyond that zone results in transition to jerk-right waveforms, with the slow phases accelerating towards the neutral zone; left lateral gaze transitions to jerk-left waveforms. Also note that the size of the jerk nystagmus grows as the eye goes more laterally; this represents a "medium" null broadness setting so the gaze-angle amplitude variation is moderate (as shown in Figure 4). In all simulations, the voluntary and intrinsic saccades work in concert to acquire and foveate the target. For large target steps, it took a greater than normal amount of time for the eye to arrive on target, also consistent with our findings in target acquisition time (Wang \& Dell'Osso, 2007).

\section{Discussion}

When the behavioral OMS model was first published in 2004 (Jacobs \& Dell'Osso, 2004), it simulated the ocular motor responses of INS patients with Pfs and PPfs waveforms and demonstrated that our hypothesis for the generation of pure pendular (P), Pfs, and PPfs waveforms could be realized by a functionally normal OMS with accurate responses to a broad variety of visual stimuli. Although jerk INS is the simplest waveform to simulate using a number of possible methods, we did not hypothesize a mechan-

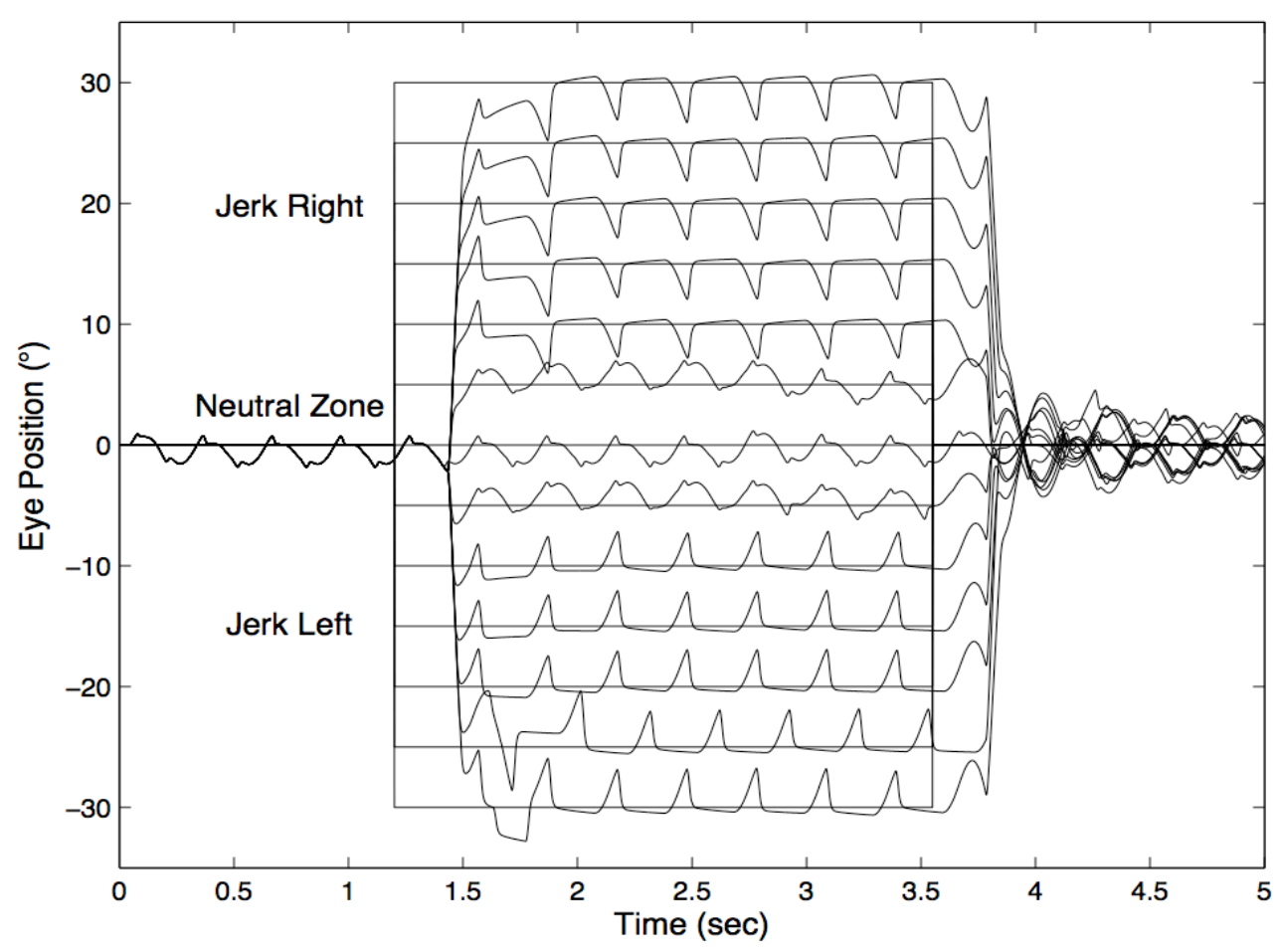

Figure 11. OMS Model simulation of an INS subject with a $\pm 5^{\circ}$, primary-position neutral zone, and a medium null broadness; jerk right waveforms occur spontaneously in right lateral gaze, jerk left in left lateral gaze. Note the accurate target foveations at all gaze angles (despite differences in INS amplitudes, waveforms, and occasional bias shifts) and the longer foveation periods common in jerk with extended foveation waveforms. 
ism at that time because such a mechanism would need to allow easy transitions with pendular waveforms when gaze is shifted. In this study, we did not have to add a separate mechanism for jerk waveforms to the current version of the OMS model because, in agreement with observations and accurate eye-movement recordings on inattention and waveform transition, the pendular and jerk waveforms of INS are derived from the same underlying mechanism, i.e., an undamped smooth pursuit subsystem. Thus, for the first time, a model has been built based on this unifying hypothesis that simulates both pendular and jerk INS behaviors, with easy, automatic transitions from one to the other.

Since the gaze-angle variations in INS resemble those of FMNS, we used the same Alexander's law input to simulate the variation of IN waveforms across the whole visual field. The behavioral output of the OMS model at different gaze angles demonstrated the effectiveness of this hypothesis. Alexander's-law imbalance (possibly asymmetric in some subjects) produced by improper calibration of the vestibular system is hypothesized to cause the INS gaze-angle variation.

The Alexander's-law effects on INS amplitude can be used in future versions of the model to modulate other INS waveforms and control the idiosyncratic transitions between pendular and jerk waveforms. The effects of inattention on INS waveforms can also be incorporated into the model, through the same modulating gain in the $\mathrm{PMC}+$ circuit.

The conception of a resettable PMC+ is in accordance with the observations about inattention discussed above. The fast phases as shown in the recordings truncate the underlying pendular component; the delaying and inhibiting of the fast phases, spontaneously or due to inattention, reveals that pendular oscillation. The resetting of an oscillation (i.e., dumping the energy) in a short amount of time is not a new concept in the ocular motor system. An eye-velocity storage mechanism has been postulated in the vestibulo-optokinetic system to account for the prolongation of vestibular nystagmus (VN) and the occurrence of optokinetic after-nystagmus (OKAN) (Kustov \& Robinson, 1995). Presentation of a subject-stationary full-field surround during VN and OKAN rapidly reduces activity related to eye velocity of the storage mechanism. This decrease in activity occurs with a shorter time constant compared to that in control trials, it has been called "dumping" (Raphan, Matsuo, \& Cohen, 1979). The PMC+ resetting is also reasonable in engineering terms. A damping circuit needs to be discharged with all its energy storing devices in order to be reset and restarted; this discharging takes time, which is consistent with the fact that our resetting signal from the fast phase motor command had to be prolonged. After the "dumping", it is also critical to restart the oscillation in a timely fashion; this is achieved by the Alexander's law output which governs the sign of the initial condition in the PMC+ resettable neural integrator. We hypothesize that the long foveation times seen in patients with Jef waveforms emerged from, and reflect, the time needed to reset the pendular oscillation and that varying that reset time allows simulation of idiosyncratic foveation-period durations.

Incidentally, we also found that altering the fast-phase scale (e.g., an inaccurate estimation of required fast-phase size) would transition the waveform from jerk to pseudocycloid. The emergence of this common INS waveform further supported the hypothesis that most of the jerk INS waveforms, although having different 
apparent morphology, originate from the same underlying pendular oscillation.

The hypothesis of a resettable slow NI is based on its behavioral input and output. Given a unidirectional signal with a DC value as an input, the slow NIs will constantly integrate if they are not periodically reset. In normals or INS subjects with pendular waveforms, this is not problematic because the slip-velocity input to the slow IN always straddles zero. In the case of FMNS simulations (Dell'Osso \& Jacobs, 2001), this also caused no problem because the velocity input to the fast NI comes from a tonic imbalance, not from the pursuit system. We hypothesized that although the slow NI does not integrate the saccadic inputs, they are still used as reset signals for the velocity input signal from the pursuit system. Physiologically, the groups of slow NI neurons and fast NI neurons are located in adjacent regions (Ugolini et al., 2006); it is possible that the slow NI neurons are connected through interneurons that enable its resetting.

Simulations with various target inputs confirmed the robustness of this unified model. Compared to most patient data, the model outputs had less cycle-to-cycle variation. This was because this model was constructed under the assumption that this oscillating OMS is otherwise healthy, i.e., no afferent deficits are present and there is little "noise" in the many calculations made by the OMS. Neither is usually true in the case of most INS patients. Also, the abilities of gaze holding and generating accurate, consistent foveating fast phases differ greatly in patients, which explains the larger idiosyncratic differences in beat-to-beat accuracy, even for patients with the same types of nystagmus waveforms.

The step-input responses shown in Figure 11 exhibit the model's ability to produce jerk waveforms with accelerating slow phases towards the neural zone (which, in this case, is at primary position). This is a property consistently found in INS eye-movement recordings; our model is the first model that successfully simulates this pathognomonic characteristic.

The values of saccadic latency in the simulations are also in agreement with human data. Eye-movement data analysis from patients with PPfs, jerk, and dual jerk waveforms showed that nystagmus subjects had a slightly higher latency than normal subjects for generating voluntary saccades (Wang \& Dell'Osso, 2007). Average values for normal subjects are $\sim 250 \mathrm{~ms}$ (Leigh \& Zee, 2006). Depending on which time of the cycle that the stimulus change occurred, the time needed to arrive at the target varied. This model used the normal subjects' average value of $\sim 250 \mathrm{~ms}$ as the saccade latency. It can easily be altered to match the value corresponding to the performance of an individual nystagmus subject.

\section{Conclusion}

This study and resulting model strongly support the hypothesis that both pendular and jerk waveforms can be generated by the same pursuit-system instability and that most INS waveforms are due to a loss of pursuit-system damping. Furthermore, the model is that of a normal OMS whose underdamped smooth pursuit system has been made undamped with no additional functional blocks added to accomplish the INS simulation. Thus, the model demonstrates the robust ability of the normal OMS to accurately maintain its gaze shifting and gaze holding functions despite an internal oscillation. By merely specifying the "null" point (the intersection of the Alexander's law lines), the sharpness of the "null"(the slopes of those lines), and the type of waveform transition from "neutral zone" to lateral gaze (e.g., all pendular, pendular to jerk, etc.), the characteristics of any spe- 
cific subject can be matched and the effects of therapeutic intervention, predicted.

Future work will simulate idiosyncratic foveation quality in INS with different durations of foveation time; the model will be tested on pursuit and more complex inputs; the transitional periods among all the waveforms will be made to occur more smoothly in the model. Simulating OMS dysfunction (e.g., INS), by otherwise normal behavioral models, continues to provide valuable insight into the functional structure of the OMS under both normal and pathological conditions.

\section{Acknowledgements}

This work was supported by the Office of Research and Development, Medical Research Service, Department of Veterans Affairs and completed as partial fulfillment for the PhD degree in Biomedical Engineering of Dr. Wang.

\section{Note}

Upon publication of this paper, version 1.5 of our Behavioral Ocular Motor System Model will be downloadable from our web site, www.omlab.org.

\section{References}

Abel, L. A., Dell'Osso, L. F., \& Daroff, R. B. (1978). Analog model for gaze-evoked nystagmus. IEEE Transactions of Biomedical Engineering, $B M E(25), 71-75$.

Abel, L. A., Dell'Osso, L. F., Schmidt, D., \& Daroff, R. B. (1980). Myasthenia gravis: Analogue computer model. Experimental Neurology, 68, 378389.

CEMAS_Working_Group. (2001). A National Eye Institute Sponsored Workshop and Publication on The Classification of Eye Movement Abnormalities and Strabismus (CEMAS). In The National Eye Institute Publications (www.nei.nih.gov). Bethesda, MD: National Institutes of Health, National Eye Institute.
Dell'Osso, L. F. (2002a). A hypothetical fixation system capable of extending foveation in congenital nystagmus. J Neuro-Ophthalmol, 22, 185-186.

Dell'Osso, L. F. (2002b). Nystagmus basics. Normal models that simulate dysfunction. In G. K. Hung \& K. J. Ciuffreda (Eds.), Models of the Visual System (pp. 711-739). New York: Kluwer Academic / Plenum Publishers.

Dell'Osso, L. F. (2006). Biologically relevant models of infantile nystagmus syndrome: the requirement for behavioral ocular motor system models. Seminars in Ophthalmology, 21(2), 71-77.

Dell'Osso, L. F., \& Daroff, R. B. (1975). Congenital nystagmus waveforms and foveation strategy. Documenta Ophthalmologica, 39, 155-182.

Dell'Osso, L. F., \& Daroff, R. B. (1976). Braking saccade--A new fast eye movement. Aviation Space Enviroknmental Medicine, 47, 435-437.

Dell'Osso, L. F., \& Daroff, R. B. (1981). Clinical disorders of ocular movement. In B. L. Zuber (Ed.), Models of Oculomotor Behavior and Control (pp. 233-256). West Palm Beach: CRC Press Inc.

Dell'Osso, L. F., Flynn, J. T., \& Daroff, R. B. (1974). Hereditary congenital nystagmus: An intrafamilial study. Archives of Ophthalmology, 92, 366374.

Dell'Osso, L. F., \& Jacobs, J. B. (2001). A normal ocular motor system model that simulates the dualmode fast phases of latent/manifest latent nystagmus. Biological Cybernetics, 85, 459-471.

Dell'Osso, L. F., \& Jacobs, J. B. (2002). An expanded nystagmus acuity function: intra- and intersubject prediction of best-corrected visual acuity. Documenta Ophthalmologica, 104, 249-276.

Dell'Osso, L. F., Weissman, B. M., Leigh, R. J., Abel, L. A., \& Sheth, N. V. (1993). Hereditary congenital nystagmus and gaze-holding failure: The role of the neural integrator. Neurology, 43, 1741-1749.

Dell'Osso, L. F., \& Wang, Z. I. (2008). Extraocular proprioception and new treatments for infantile nystagmus syndrome. In C. Kennard \& R. J. Leigh (Eds.), Progress in Brain Research, Vol 171, Using Eye Movements as an Experimental Probe of Brain Function, A Symposium in Honour of Jean Büttner-Ennever (pp. 67-75): Elsevier.

Doslak, M. J., Dell'Osso, L. F., \& Daroff, R. B. (1982). Alexander's law: A model and resulting study. Annals of Otology Rhinology and Laryngology, 91, 316-322.

Jacobs, J. B. (2001). An Ocular Motor System Model that Simulates Congenital Nystagmus, Including 
Braking and Foveating Saccades. (Ph.D. Dissertation). Case Western Reserve University, Cleveland.

Jacobs, J. B., \& Dell'Osso, L. F. (2004). Congenital nystagmus: hypothesis for its genesis and complex waveforms within a behavioral ocular motor system model. Journal of Vision, 4(7), 604-625.

Jacobs, J. B., Dell'Osso, L. F., \& Erchul, D. M. (1999). Generation of braking saccades in congenital nystagmus. Neuro-Ophthalmology, 21, 83-95.

Kustov, A. A., \& Robinson, D. L. (1995). Modified saccades evoked by stimulation of the Macaque superior colliculus account for properties of the resettable integrator. Journal of Neurophysiology, 73, 1724-1728.

Leigh, R. J., \& Zee, D. S. (2006). The Neurology of Eye Movements, Edition 4 (Contemporary Neurology Series). New York: Oxford University Press.

Raphan, T., Matsuo, V., \& Cohen, B. (1979). Velocity storage in the vestibuloocular reflex arc (VOR). Experimental Brain Research, 35, 229-248.

Robinson, D. A., Gordon, J. L., \& Gordon, S. E. (1986). A model of smooth pursuit eye movements. Biological Cybernetics, 55, 43-57.

Robinson, D. A., Zee, D. S., Hain, T. C., Holmes, A., \& Rosenberg, L. F. (1984). Alexander's law: Its behavior and origin in the human vestibuloocular reflex. Annals of Neurology, 16, 714-722.
Ugolini, G., Klam, F., Doldan Dans, M., Dubayle, D., Brandi, A. M., Buttner-Ennever, J., \& Graf, W. (2006). Horizontal eye movement networks in primates as revealed by retrograde transneuronal transfer of rabies virus: differences in monosynaptic input to "slow" and "fast" abducens motoneurons. Journal of Comparative Neurology, 498(6), 762-785.

Wang, Z., Dell'Osso, L. F., Zhang, Z., Leigh, R. J., \& Jacobs, J. B. (2006). Tenotomy does not affect saccadic velocities: Support for the "smallsignal" gain hypothesis. Vision Research, 46, 2259-2267.

Wang, Z. I. (2008). A unifying hypothesis for the multiple waveforms of infantile nystagmus and their idiosyncratic variation with gaze angle and therapy. (Ph.D. Dissertation). Case Western Reserve University, Cleveland.

Wang, Z. I., \& Dell'Osso, L. F. (2007). Being "slow to see" is a dynamic visual function consequence of infantile nystagmus syndrome: Model predictions and patient data identify stimulus timing as its cause. Vision Research, 47(11), 1550-1560. 